INL/EXT-17-43255 Rev. 0

September 2017

\title{
Progress update on lower length scale research and development on U3Si2 fuel and FeCrAl cladding
}

\author{
Y. Zhang \\ L. Aagesen \\ B. Beeler \\ C. Jiang \\ K. Ahmed \\ J. $Y u$ \\ D. Schwen \\ D. Andersson \\ M. Baskes \\ M. Cooper \\ E. Martinez
}




\section{NOTICE}

This information was prepared as an account of work sponsored by an agency of the U.S.

Government. Neither the U.S. Government nor any agency thereof, nor any of their employees, makes any warranty, express or implied, or assumes any legal liability or responsibility for any third party's use, or the results of such use, of any information, apparatus, product, or process disclosed herein, or represents that its use by such third party would not infringe privately owned rights. The views expressed herein are not necessarily those of the U.S. Nuclear Regulatory Commission. 
INL/EXT-17-43255 Rev. 0

Progress update on lower length scale research and development on U3Si2 fuel and FeCrAl cladding

\author{
Y. Zhang \\ L. K. Aagesen \\ B. Beeler \\ C. Jiang \\ K. Ahmed \\ J. $Y u$ \\ D. Schwen \\ D. Andersson * \\ M. Baskes" \\ M. Cooper" \\ E. Martinez"
}

September 2017

\author{
Idaho National Laboratory \\ Fuel Modeling and Simulation Department \\ Idaho Falls, Idaho 83415 \\ "Los Alamos National Laboratory \\ Los Alamos, New Mexico 87545
}

Prepared for the

U.S. Department of Energy

Office of Nuclear Energy

Under U.S. Department of Energy-Idaho Operations Office

Contract DE-AC 07-99ID13727 


\begin{abstract}
This report summarizes the lower length scale model development and studies in regards to two accident tolerant (ATF) concepts, $\mathrm{U}_{3} \mathrm{Si}_{2}$ and FeCrAl. $\mathrm{U}_{3} \mathrm{Si}_{2}$ is proposed to replace $\mathrm{UO}_{2}$ fuel for its high thermal conductivity and higher uranium density. FeCrAl alloys are proposed to replace $\mathrm{Zr}$ based cladding for their high mechanical strength and good corrosion resistance. Before applying them in reactors, sufficient data are needed to establish their predictable in-pile performance, but are largely missing, as they are costly and time consuming to obtain by experimental approaches. Under the NEAMS ATF high-impact-problem (HIP), multiscale modeling and simulations are used to develop atomistic and mesoscale tools, obtain fundamental material properties, and assess the behavior of these two ATF concepts. The accomplishments in FY17 are briefly introduced in this report including the development of interatomic potentials, assessment of swelling in $\mathrm{U}_{3} \mathrm{Si}_{2}$, and $\mathrm{Cr}$ precipitation in $\mathrm{FeCrAl}$ alloys.
\end{abstract}




\section{Table of Contents}

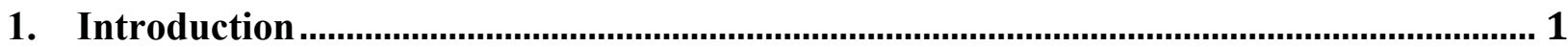

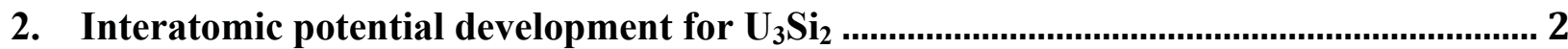

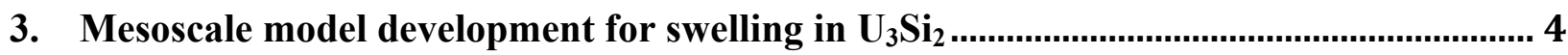

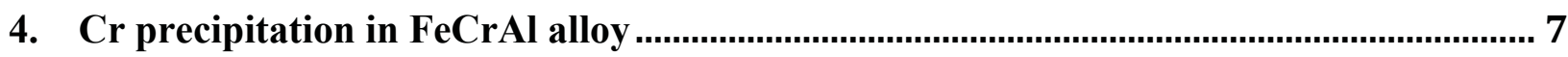

5. Thermal conductivity of uranium silicides .................................................................... 9

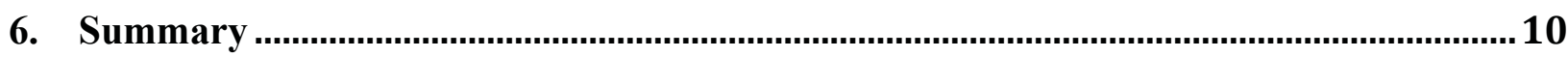

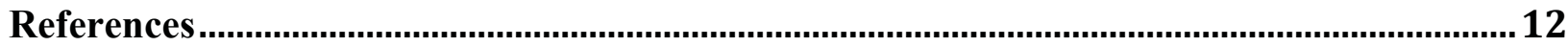




\section{Introduction}

Following the Fukushima Daiichi accident in 2011, the development of accident tolerant fuels (ATFs) has become a primary direction in nuclear fuel research and development for light water reactors (LWRs) [1]. Compared with the standard oxide fuel $\left(\mathrm{UO}_{2}\right.$ fuel with $\mathrm{Zr}$-alloy cladding), the ATFs - including both fuels and claddings - are required to have better or at least comparable performance during normal operation, and better performance in accident scenarios such as loss of coolant accidents (LOCA). The ATFs are expected to sustain a longer time than the oxide fuel under off-normal conditions and thus allow for enhanced safety margins. Two leading concepts, triuranium-disilicide $\left(\mathrm{U}_{3} \mathrm{Si}_{2}\right)$ as fuel and FeCrAl as cladding respectively, have attracted wide research and applied interests.

Compared to $\mathrm{UO}_{2}, \mathrm{U}_{3} \mathrm{Si}_{2}$ has higher thermal conductivity, which increases with increasing temperature. This allows for a lower fuel centerline temperature during operation and helps to mitigate the reduced heat exchange with coolant when an accident such as LOCA occurs. The higher $\mathrm{U}$ density may enable some new cladding concepts that would otherwise require increased enrichment limits to compensate for their neutronic penalty. These beneficial properties have made $\mathrm{U}_{3} \mathrm{Si}_{2}$ one of the leading ATF concepts being actively explored. Similarly, FeCrAl alloys are proposed to replaced $\mathrm{Zr}$ based claddings for their better corrosion resistance and high mechanical strength [2]. The high resistance to corrosion originates from the formation of an aluminum oxide layer at the outer surface which delays further oxidation. The high mechanic strength may enhance the burst margin of the cladding.

In spite of the beneficial properties, these ATF concepts need to be studied extensively to gain sufficient confidence on their in-pile and out-of-pile behaviors. The fuels should be mechanically stable. The dimensional change and property degradation during fuel operation need to be compatible with the current reactor design, and be predictable, without risks of unexpected fuel failure. Given the scarcity of available data, it is very challenging to obtain sufficient data from experiments in and out of reactors to establish predictive fuel performance models. In order to accelerate and assist ATF fuel development and the down-selection of ATF concepts, multiscale modeling and simulations similar to that for $\mathrm{UO}_{2}$ fuel and Zircaloy cladding are utilized in the NEAMS Accident-Tolerant-Fuel project to improve the fundamental understanding and to develop materials models for $\mathrm{U}_{3} \mathrm{Si}_{2}$ fuel and $\mathrm{FeCrAl}$ cladding. As these two concepts have not been extensively studied, many tools required for studies at the atomistic scale and mesoscale are yet to be developed.

Driven by the need for data and tools, lower-length-scale efforts have been made to develop atomistic to mesoscale tools, to compute materials properties, explore fundamental mechanisms that govern material behavior, and to assess fuel performance during fuel operation [3]. The progress made is briefly summarized in the following Sections, including the development of interatomic potentials, a phase field swelling model for $\mathrm{U}_{3} \mathrm{Si}_{2}$ in the MARMOT mesoscale code, and a kinetic Monte Carlo model for $\mathrm{Cr}$ precipitation in $\mathrm{FeCrAl}$ alloys. A thermal conductivity model for various uranium silicides has also been developed. 


\section{Interatomic potential development for $\mathbf{U}_{3} \mathbf{S i}_{2}$}

Understanding fuel behavior involving interfaces and/or extended defects is crucial for predicting fuel performance. To fully understand the relevant material science issues atomic scale resolution is usually required. However, the computational cost is too expensive for first principle, density functional theory calculations. An alternative choice is to use molecular dynamics simulations, which require interatomic potentials to describe the interaction between atoms and/or ions. Given that no suitable potentials for the material systems of interest existed before this project, two versions of interatomic potentials, one Tersoff type and one modified embedded-atom-method (MEAM) type, have been developed. The Tersoff potential has been documented in a previous report [4] and a conference proceeding [5]. The MEAM potential has recently been accepted for journal publication [6], and will be briefly introduced here.

The MEAM interatomic potential has been developed with particular emphasis on $\mathrm{U}_{3} \mathrm{Si}_{2}$. This has been accomplished by utilizing a pre-existing uranium potential, modifying a pre-existing $\mathrm{Si}$ potential, and fitting cross species parameters to experimental and DFT data on $\mathrm{U}_{3} \mathrm{Si}_{2}$. The potential has been utilized to investigate a variety of $0 \mathrm{~K}$ properties, including elastic constants and defects, while also being implemented to obtain high temperature properties of $\mathrm{U}_{3} \mathrm{Si}_{2}$.

\subsection{Summary of potential performance}

The formation energy of $\mathrm{U}_{3} \mathrm{Si}_{2}$ is reproduced very accurately while the volume per atom is slightly underestimated. The $a$ lattice constant is underestimated and the $c$ lattice constant is slightly overestimated. The elastic constants show varying degrees of agreement with DFT predictions. Excellent agreement is shown for $\mathrm{Gxz}, \mathrm{C}_{33}$ and $\mathrm{C}_{66}$, but significant variance is observed for $\mathrm{C}_{12}$, for example. The resulting bulk modulus, calculated via the elastic constants, is overestimated. The bulk modulus was also calculated via the Birch-Murnaghan curve, yielding a value of $134.5 \mathrm{GPa}$, which is consistent with the calculation of the bulk modulus via elastic constants. There is observed a general over-stiffness prediction of the elastic constants. The rootmean-square error over the nine calculated elastic constants is $55.0 \mathrm{GPa}$.

Single point defects (vacancy, interstitial, anti-site) were considered as a means of accommodating a change in stoichiometry. Excellent agreement is observed for the formation energy of the U2 vacancy, Si vacancy, U interstitial, Si interstitial and the U1 antisite. Reasonable agreement is observed for the U2 anti-site defect. The U1 vacancy and Si antisite formation energies are overestimated. The overestimation of the U1 vacancy likely leads to the slight overestimation of the bound Schottky defect formation energy.

In order to ensure that the potentials are not restricted to studying only the $\mathrm{U}_{3} \mathrm{Si}_{2}$ phase, other phases, both experimental and theoretical, were examined that were not included in the fitting procedure. Excellent agreement is observed for the U-rich portion of the composition range when considering the formation energy per atom. For $\mathrm{U}_{3} \mathrm{Si}$ and $\mathrm{U}_{3} \mathrm{Si}_{2}$, formation energies are nearly identical to those from experiments and DFT calculations. The lowest energy structure is the $\mathrm{U}_{3} \mathrm{Si}_{2}$ phase, which is the phase of primary interest, however this does not match the experimental or DFT results, which find the $\mathrm{USi}_{2}$ and $\mathrm{U}_{3} \mathrm{Si}_{5}$ structures to be lowest in energy, respectively. Greater variance of the formation energy per atom is observed in the Si-rich composition regime. Generally, there does exist a convex hull shape to the formation energy 
versus composition curve, which agrees with experiments and DFT. A general negative parabolic trend in the volume per atom predicted as a function of composition is observed, which matches the trends from DFT and experiments quite well. Given that only $\mathrm{U}_{3} \mathrm{Si}_{2}$ was utilized as a fitting target and $\mathrm{L} 12-\mathrm{U}_{3} \mathrm{Si}$ was utilized as a reference structure, there is considerable agreement across the entire composition spectrum when comparing MEAM predicted results to the results from DFT calculations.

The total energy and total volume of $\mathrm{U}_{3} \mathrm{Si}_{2}$ as a function of temperature were investigated (see Fig. 1). The structure is stable and behaves predictably as a function of temperature with no observed crystal structure changes or discontinuities suggesting potential instabilities. The calculated average linear thermal expansion from $200 \mathrm{~K}$ to $1200 \mathrm{~K}$ is $11.8 \times 10^{-6} \mathrm{~K}^{-1}$ for the U-Si MEAM potential, while the experimental linear thermal expansion is $14-17 \times 10^{-6} \mathrm{~K}^{-1}$. While slightly underestimated, this is remarkable agreement given that this was not included into the fitting procedure. The molar heat capacity was calculated at $400 \mathrm{~K}$ for comparison with experiments. The calculated value from the MEAM potential is $134.7 \mathrm{~J} / \mathrm{mol}-\mathrm{K}$. This quantity compares very favorably to the Dulong-Petit value of $125 \mathrm{~J} / \mathrm{mol}-\mathrm{K}$ and the experimental value of $150 \mathrm{~J} / \mathrm{mol}-\mathrm{K}$. This is considered excellent agreement to both theory and experiment.

The high temperature regime was investigated by calculating the melting point of $\mathrm{U}_{3} \mathrm{Si}_{2}$. The calculated melting point is approximately $1775 \mathrm{~K}$ for the U-Si MEAM potential. The experimental melting point is $1938 \mathrm{~K}$. Although the melting point is underestimated by approximately $150 \mathrm{~K}$, this is still considered excellent agreement.

The (100) and (001) free surfaces were also investigated at $500 \mathrm{~K}$ (see Fig. 1). The structure remains stable and retains its crystal symmetry. The energy for the (100) surface is $1.73 \mathrm{~J} / \mathrm{m}^{2}$ and the $(001)$ surface energy is $1.75 \mathrm{~J} / \mathrm{m}^{2}$. A prior DFT study investigated free surfaces in $\mathrm{U}_{3} \mathrm{Si}_{2}$, finding that the (100) surface had a surface energy of $1.48 \mathrm{~J} / \mathrm{m}^{2}$, while the (001) surface had a surface energy of $1.43 \mathrm{~J} / \mathrm{m}^{2}$. A variance of less approximately $0.3 \mathrm{~J} / \mathrm{m}^{2}$ is considered very good agreement. A void was also studied to obtain an average surface energy. Voids of up to $6.4 \mathrm{~nm}$ in diameter were explored. No void collapse is observed, no crystal structure collapse or distortion is observed, but minimal void surface reconstruction does occur. The representative void surface energy is $1.69 \mathrm{~J} / \mathrm{m}^{2}$ for the U-Si MEAM potential.

In order to test the ability of the potential to model radiation damage, a $1 \mathrm{keV}$ cascade is investigated. It is observed that the crystal structure is stable and there exists a thermal spike and subsequent annealing stage, as would be expected. At 0.4 ps after cascade initiation, WignerSeitz analysis shows that there exist 35 Frenkel pairs; there exist 22 Frenkel pairs after 12 ps; there exist 17 Frenkel pairs after $1 \mathrm{~ns}$. Looking strictly at the uranium sublattice after $1 \mathrm{~ns}$, there are 16 Frenkel pairs. Looking strictly at the silicon sublattice, there are 12 Frenkel pairs. It can be concluded that this potential is displaying reasonable behavior under irradiation and the nature and number of defects can be readily determined.

Given the complex nature of the crystal structure of $\mathrm{U}_{3} \mathrm{Si}_{2}$, the inherent difficulties associated with the development of atomic potentials for pure uranium, let alone uranium-alloys, a suitable interatomic potential to describe the U-Si system has been developed. 

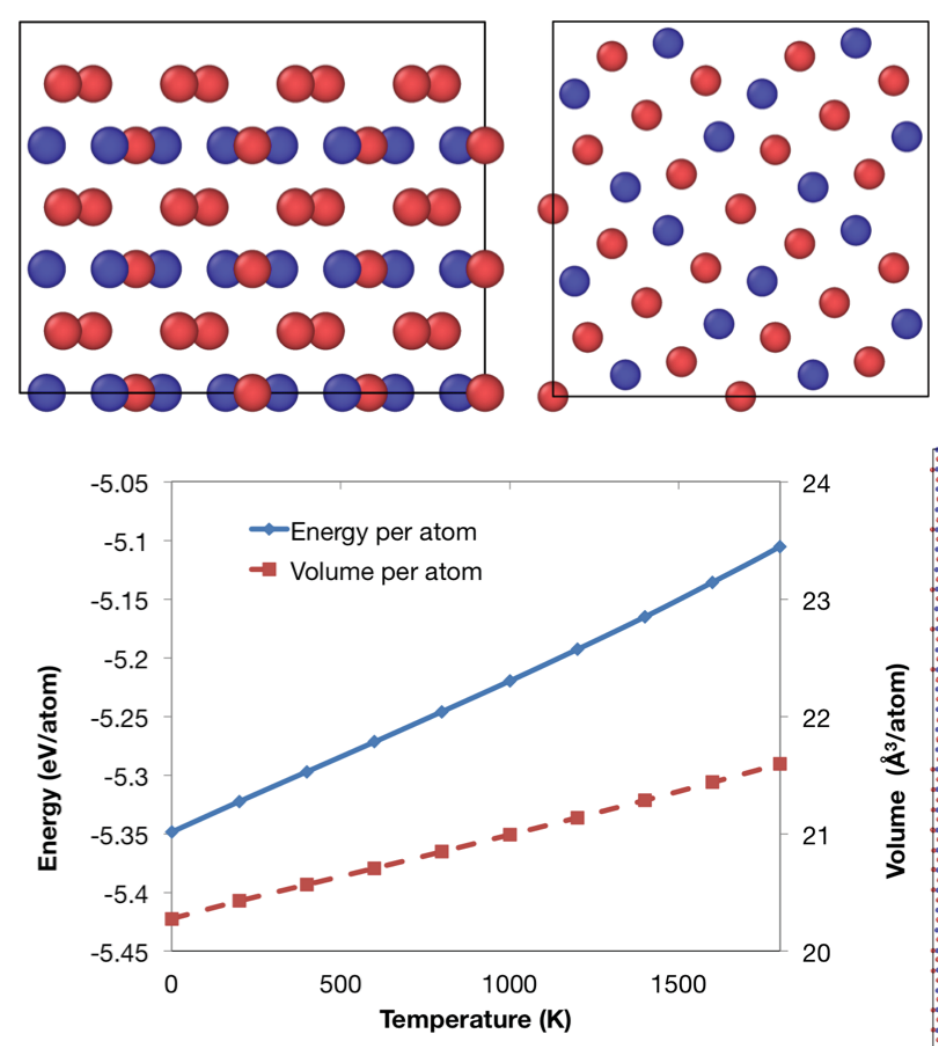

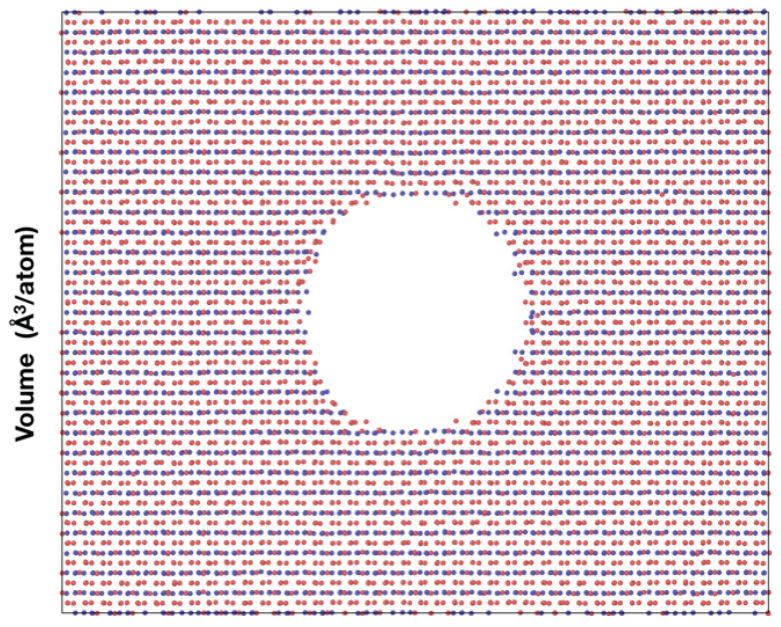

Figure 1. Top left: Crystal structure of $\mathrm{U}_{3} \mathrm{Si}_{2}$. Bottom left: Energy and volume per atom of $\mathrm{U}_{3} \mathrm{Si}_{2}$ as a function of temperature. Top right: (100) and (001) free surfaces or $\mathrm{U}_{3} \mathrm{Si}_{2}$ at $500 \mathrm{~K}$. Bottom right: $5 \mathrm{~nm}$ radius void at $500 \mathrm{~K}$ in $\mathrm{U}_{3} \mathrm{Si}_{2}$.

\subsection{Planned implementation of the U-Si potential}

The U-Si MEAM potential will be utilized to investigate a variety of phenomena within the next fiscal year. This includes grain boundary energy and surface energy variation as a function of temperature, radiation damage behavior, irradiation enhanced diffusion, xenon thermal and irradiation enhanced diffusion in bulk and along grain boundaries, and various properties of equilibrium, over- and under-pressurized bubbles.

\section{Mesoscale model development for swelling in $\mathbf{U}_{3} \mathbf{S i}_{2}$}

Due to the lower operating temperature of $\mathrm{U}_{3} \mathrm{Si}_{2}$ than that of $\mathrm{UO}_{2}$, there is a possibility that no gas release will occur until very high burn-up. As all fission gases are contained in the fuel, significant fuel swelling may possibly occur, alternating fuel dimensions and possibly causing severe fuel cladding mechanical interaction. Therefore, a good assessment of swelling is needed for $\mathrm{U}_{3} \mathrm{Si}_{2}$ to be developed further as an ATF concept. In FY17, a mesoscale model is developed in MARMOT at the grain level to quantify fuel swelling in $\mathrm{U}_{3} \mathrm{Si}_{2}$. The model and the preliminary assessment will be documented in a separate report [7] with more details. A brief introduction is given here. 
To estimate swelling in nuclear fuel, a commonly used approach is to assume that it is equal to the volume fraction of fission gas bubbles in the fuel microstructure. To predict swelling in $\mathrm{U}_{3} \mathrm{Si}_{2}$, a phase-field model to simulate the evolution of the fission gas bubble microstructure was developed. From the simulated microstructure, the volume fraction of bubbles can be calculated to estimate swelling. The new phase-field model is a multi-phase, multi-order parameter extension of the grand-potential phase field model [8] and is implemented in MOOSE/MARMOT. This model allows an arbitrary number of phases and grains of each phase to be included, allowing for simulations of polycrystalline fuel microstructures with inter- and intra-granular bubbles. This model removes the excess chemical energy from the interfacial regions, simplifying parameterization of the interfacial energy. The decoupling of chemical energy and interfacial energy also allows interfacial thickness to be made arbitrarily wide (subject to adequately resolving the microstructural features of interest). These wider interfaces allow the use of a coarser mesh in simulations, increasing computational efficiency. The model is similar in this respect to the existing Kim-Kim-Suzuki phase-field model [9], but it does not require the use of additional phase concentration variables, which further improves computational efficiency. Finally, in contrast to many existing multi-order parameter phase field models, in the new model, interfaces between two phases are stable with respect to the formation of additional phases.

In addition to the local phase information, the phase-field model also tracks the local concentrations of U-site vacancies and fission gas atoms on $U$ lattice sites. The production of these defect species is incorporated in the evolution equations using source terms. The fission gas atoms are assumed to be all Xe atoms since they are the predominant insoluble fission gas product. To incorporate fission gas bubble pressure into phase-field models of fission gas bubbles, an eigenstrain approach has typically been used in the past. However, the eigenstrain approach requires tedious calculations of the Eshelby tensor for each pressure, and does not give a hydrostatic pressure for intergranular bubbles. Therefore, we have developed implemented a new approach in which the gas bubble hydrostatic stress state is specified directly and interpolated for each phase using the order parameters. A base class ComputeExtraStressBase was added to the MOOSE framework to implement this approach. For a constant gas pressure, the material ComputeExtraStressConstant was added. This allows the effect of fission gas bubble pressure on microstructural evolution to be evaluated.

Total swelling in $\mathrm{U}_{3} \mathrm{Si}_{2}$ was calculated using the phase-field model. The swelling in the intergranular and intragranular regions was considered independently, and the total swelling was determined using a volume-fraction average of the contributions of the two regions. Simulations were parameterized using DFT and MD simulations with the MEAM interatomic potential developed for the ATF-HIP in FY17. Simulations of the intragranular region were conducted in $2 \mathrm{D}$ and included the effect of re-solution as a reaction term in the Allen-Cahn equation. The microstructure is shown in Fig. 2(a), and swelling (as measured by volume fraction of the bubble phase) is shown in Fig. 2(c). Swelling in the intergranular region was simulated in 3D, assuming a single intergranular bubble between two grains. It was assumed that there exists a denuded zone free of intragranular bubbles a distance of $160 \mathrm{~nm}$ on either side of the grain boundary. The bubble initial size and density was based on experimental observations in $\mathrm{UO}_{2}$. The structure of the intergranular bubble is shown in Fig. 2(b), and resultant swelling is shown in Fig. 2(d). From the swelling predictions of the two regions, the total swelling is calculating using a volume 
fraction average of the two regions. The volume fraction is calculated assuming the $\mathrm{U}_{3} \mathrm{Si}_{2}$ has a grain structure approximated as truncated octahedra, a space-filling 14-sided polyhedron. The grain size is 5 microns, and the intragranular region is assumed to be smaller by $160 \mathrm{~nm}$ in each direction based on the width of the denuded zone. Using these approximations, the volume fraction of the intragranular region was calculated to be $76 \%$. The total swelling under these assumptions is shown in Fig. 2(e).

The previously described phase-field simulations, parameterized by lower length scale calculations, represent a large step forward in simulating fission gas bubble evolution in a physical way and estimating swelling. However, several model parameters have a high degree of uncertainty, and should be improved to increase the accuracy of these simulations. Although the production of fission gas atoms can be reasonably approximated based on the expected fission rate density and fission yield, the model relies on the assumption that the net rate of vacancy production is ten times that of gas production. This assumption about the net vacancy production rate has a strong influence on the swelling rate. However, in reality many more interstitialvacancy pairs are created, but many rapidly recombine, and there is a net production of vacancies because the less tightly bound interstitial atoms diffuse more rapidly to sinks such as dislocation loops and grain boundaries. To improve the accuracy of the model, two possible approaches are described. The first would be to track interstitial concentration in the phase field model, to include interstitial production in addition to vacancy production, and to include the presence of sink terms for both interstitial and vacancies. However, the parameterization of the sink strengths would likely be challenging to do in a physical way. Another possible approach would be to use atomistic modeling techniques to physically parameterize the net vacancy production rate.

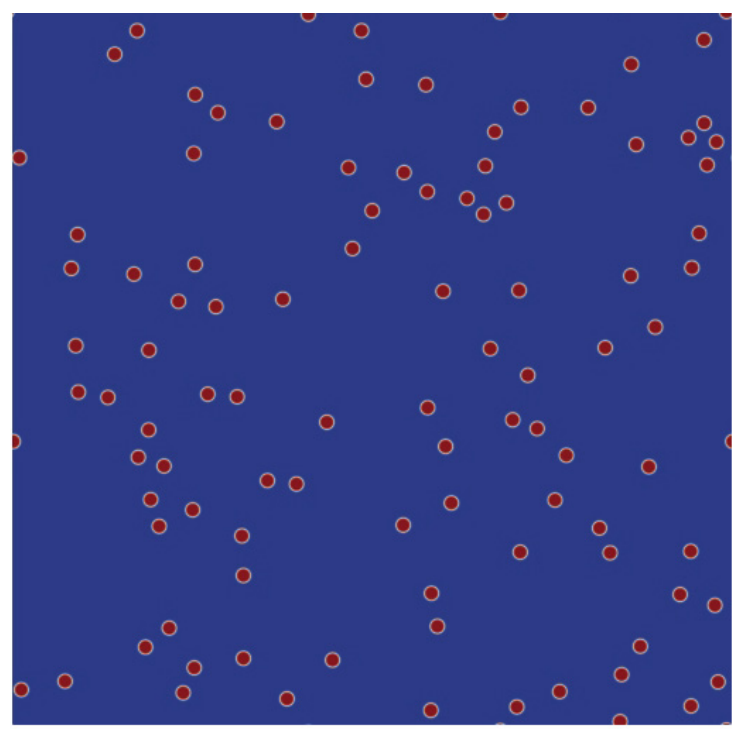

(a)

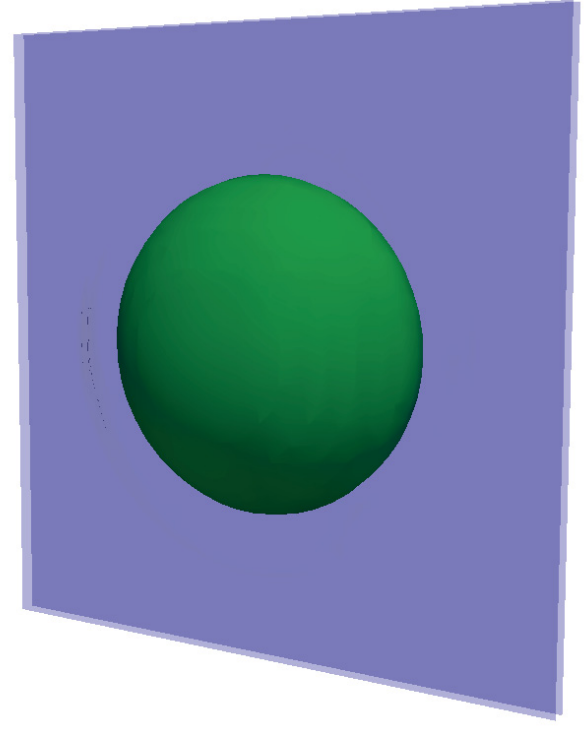

(b) 


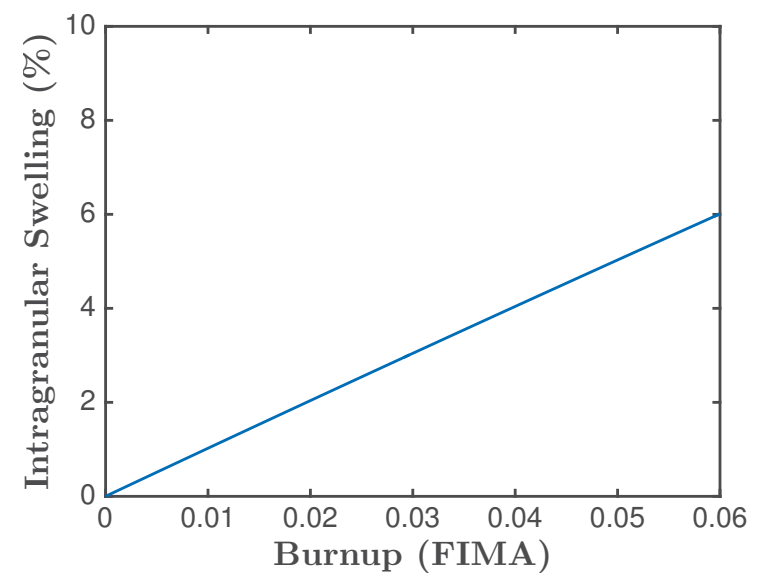

(c)

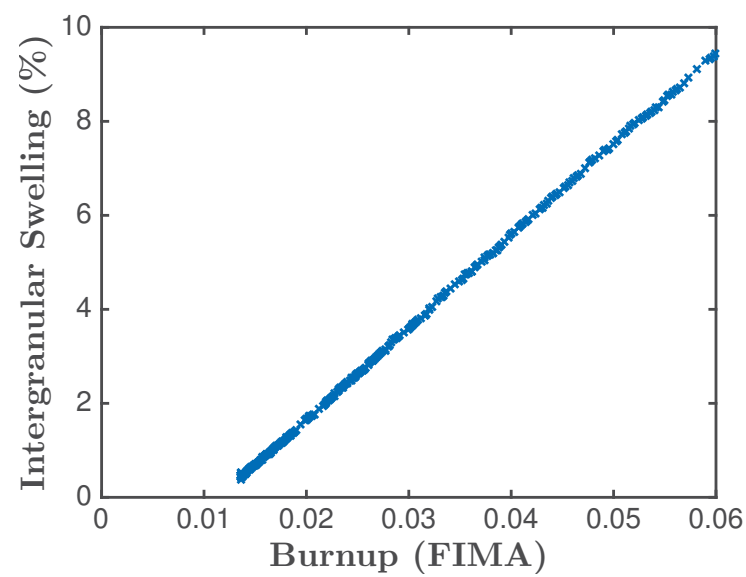

(d)

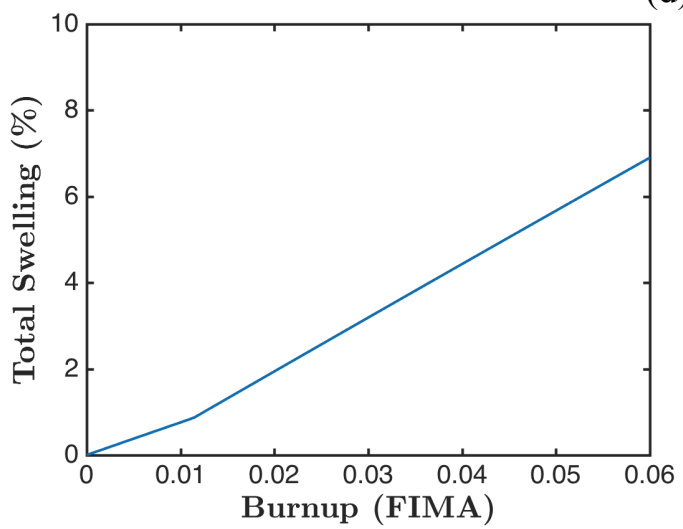

(e)

Figure 1. (a) Simulation of intragranular bubbles. (b) Simulation of intergranular bubble. (c) Swelling in the intragranular region, as determined by the volume fraction of bubbles. (d) Swelling in the intergranular region, as determined by the volume fraction of the intergranular bubble. (e) Total swelling, as determined from a volume-fraction weighted average of the intragranular and intergranular bubble regions.

\section{Cr precipitation in FeCrAl alloy}

FeCrAl alloy has been identified as a possible cladding material for accident tolerant fuels due to its excellent corrosion resistance via the formation of a protective, slow growing alumina surface layer. However, the precipitation of $\mathrm{Cr}$-rich $\alpha^{\prime}$ clusters in $\mathrm{FeCrAl}$ can lead to degraded mechanical properties such as the well known " $475^{\circ} \mathrm{C}$ embrittlement", and should therefore be avoided to ensure cladding integrity. In FY16, a lattice kinetic Monte Carlo (LKMC) model was developed for $\mathrm{FeCr}$ binary alloys. Due to the assumption of constant bond energies, the previous model cannot correctly reproduce the low-temperature part of the $\mathrm{Fe}-\mathrm{Cr}$ phase diagram. It is also not possible to model the anomalous ordering tendency and repulsive interactions between $\mathrm{Cr}$ atoms in $\alpha \mathrm{Fe}$ at the dilute concentration regime using constant bond energies. To address these issues, a new local-environment-dependent bond energy formalism has been developed for $\mathrm{Fe}-\mathrm{Cr}$ in FY17 that can simultaneously reproduce the negative mixing energy at low $\mathrm{Cr}$ concentrations and the positive mixing energy at high $\mathrm{Cr}$ concentrations, and the consequent large solubility of $\mathrm{Cr}$ in bcc Fe at low temperatures. Furthermore, the LKMC model has been extended to include 
$\mathrm{Al}$ to enable the assessment of $\mathrm{Cr}$ precipitation tendency in ternary $\mathrm{FeCrAl}$ alloys under thermal aging. All parameters of this model are derived by fitting to DFT calculated data such as the total energy change associated with a nearest-neighbor vacancy jump, the binding energy for atomatom or atom-vacancy interactions, migration barriers for vacancy jump, and the mixing energy of random alloys. More details of the model have been documented in a recent report [10].

For $\mathrm{KMC}$ simulations of $\mathrm{Cr}$ precipitation in bcc $\mathrm{FeCrAl}$, we employ the rejection-free residence time algorithm and transition state theory widely used in the literature for studying the precipitation of solute elements in bcc Fe [11-16]. A vacancy-mediated diffusion mechanism is assumed, which is valid for thermal aging conditions. Note that, under irradiation, diffusion of chemical species can be greatly accelerated due to super-saturation of point defects (vacancies and interstitials) created by collision cascades, which is called radiation-enhanced diffusion. Diffusion of interstitials or vacancies towards defect sinks can also lead to redistribution of solute concentrations, so-called radiation-induced segregation. For simplicity, such effects are not considered in this work.

Driven by the local-environment-dependent bond energy model developed in this work, KMC simulations have been performed to study $\alpha^{\prime}$ precipitation in bcc $\mathrm{Fe}-\mathrm{Cr}$ and $\mathrm{Fe}-\mathrm{Cr}-\mathrm{Al}$ alloys under thermal aging conditions assuming equilibrium vacancy concentration. All simulations start from an initially homogenized simulation cell containing a single vacancy that can exchange with one of its eight first nearest neighbor atoms. In agreement with experiments [17], the present $\mathrm{KMC}$ simulations predict no $\mathrm{Cr}$ precipitation in $\mathrm{Fe}-10 \mathrm{Cr}-8 \mathrm{Al}$ alloy and $\mathrm{Cr}$ precipitation in $\mathrm{Fe}-21 \mathrm{Cr}-10 \mathrm{Al}$ at $500^{\circ} \mathrm{C}$, as shown in Figure 3. It can be seen that the short-range order (SRO) parameter for $\mathrm{Fe}-10 \mathrm{Cr}-8 \mathrm{Al}$ alloy quickly reaches its equilibrium value, which is negative due to the ordering of $\mathrm{Cr}$ atoms. In contrast, for $\mathrm{Fe}-21 \mathrm{Cr}-10 \mathrm{Al}$, the $\mathrm{SRO}$ parameter slowly increases with time. The positive SRO is an indication of phase separation. Here, the phase separation process is much slower than ordering since the former requires long-range diffusion of $\mathrm{Cr}$ atoms. In Figure 3, KMC results for binary $\mathrm{Fe}-20 \mathrm{Cr}$ alloy are also shown. It can be seen that the SRO parameter of the Fe-20Cr alloy initially rapidly increases with time, which is due to the nucleation and growth of $\alpha^{\prime}$ particles. For longer time ( $>2000$ hours), the curve becomes almost flat, indicating the coarsening stage when small $\alpha^{\prime}$ particles grow into bigger ones. After 5000 hours of thermal aging, a large $\alpha^{\prime}$ cluster is formed near the center of the simulation cell. The KMC predicted $\mathrm{Cr}$ concentrations of $\alpha$ and $\alpha^{\prime}$ phases are in good agreement with the values determined by the 3D-APT experiments of Novy et al. [18]. Such an agreement is remarkable considering the intrinsic uncertainties in both modeling and experiments.

In summary, a new local-environment-dependent bond energy model has been developed for FeCrAl during FY17. This new model can simultaneously describe the ordering tendency (repulsive $\mathrm{Cr}-\mathrm{Cr}$ interaction) in the dilute solid solution phase and the phase separating tendency (attractive $\mathrm{Cr}-\mathrm{Cr}$ interactions) at high $\mathrm{Cr}$ concentrations in $\mathrm{Fe}-\mathrm{Cr}$-based alloys. For $\mathrm{Fe}-20 \mathrm{Cr}$ alloy, results from the present $\mathrm{KMC}$ simulations show good agreement with available experiments in the literature. Importantly, present $\mathrm{KMC}$ simulations predict $\alpha^{\prime}$ precipitation in Fe-21Cr-10Al alloy but not in Fe-10Cr-8Al alloy, which is also in complete agreement with experiments. Despite its success, one limitation of the present $\mathrm{KMC}$ model is that it is only applicable to thermal aging conditions. In future studies, the $\mathrm{KMC}$ model will be extended to incorporate the effects of irradiation such as radiation-driven mixing on $\mathrm{Cr}$ precipitation in FeCrAl. Mixing is believed to destabilize the $\alpha^{\prime}$ particles and thus has an important impact on 
microstructural evolution.

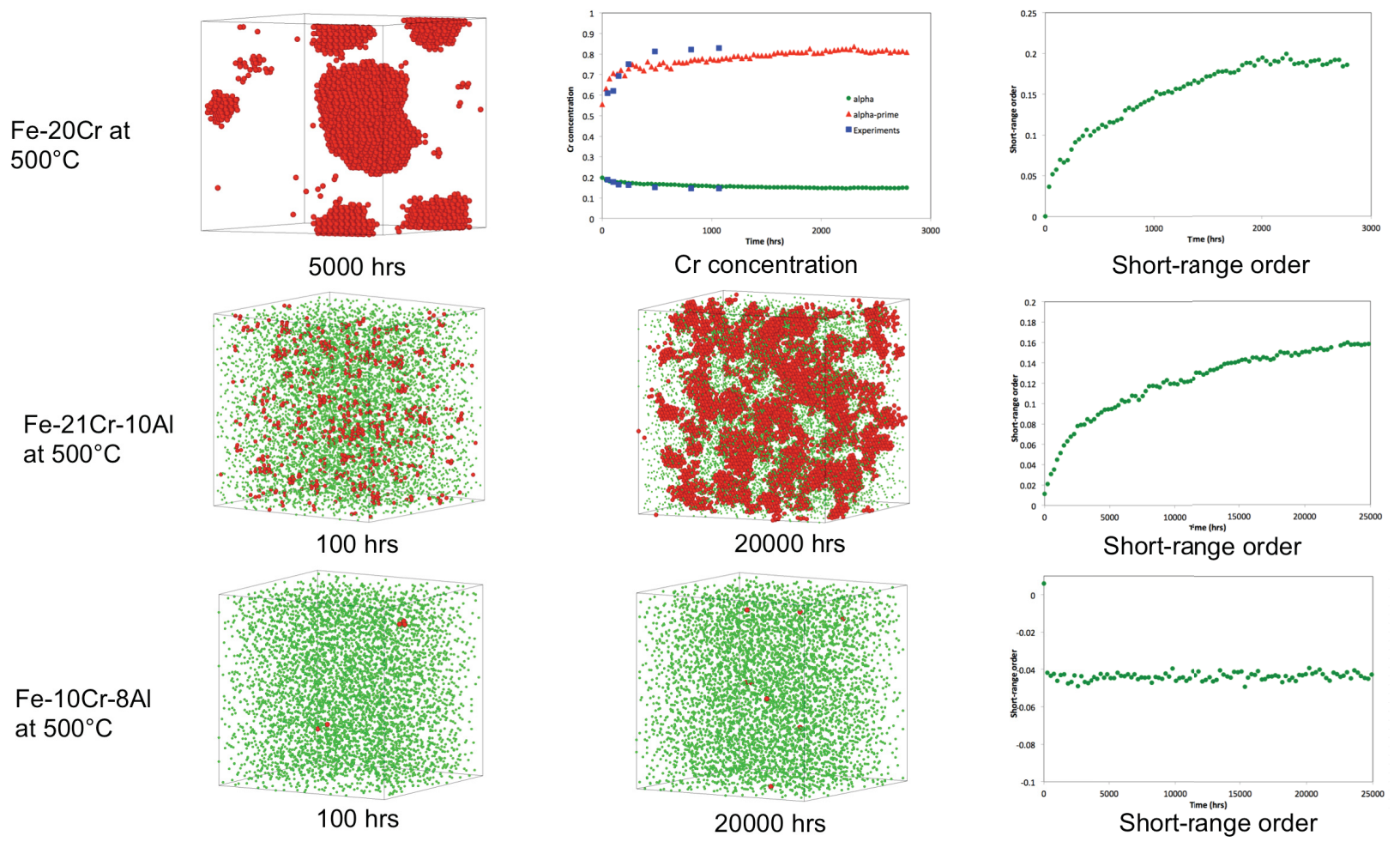

Figure 3. KMC simulation of $\mathrm{Cr}$ precipitation in bcc Fe-20Cr, Fe-21Cr-10Al and Fe-10Cr-8Al alloys at $773 \mathrm{~K}$. Green spheres denote $\mathrm{Al}$ atoms. Red spheres denote $\mathrm{Cr}$ atoms in $\alpha^{\prime}$ clusters. For clarity of visualization, all $\mathrm{Cr}$ atoms in the solid solutions and all $\mathrm{Fe}$ atoms are not shown. For Fe-20Cr, data from APT experiments are also shown for comparison.

\section{Thermal conductivity of uranium silicides}

The primary beneficial property of $\mathrm{U}_{3} \mathrm{Si}_{2}$ over $\mathrm{UO}_{2}$ is the higher thermal conductivity. While under irradiation during operation, decay in thermal conductivity may take place due to the production of lattice defects and fission products, porosity, and possible phase transformation. In the previous year, a model describing the conductivities in various silicides has been proposed [19], with extra terms accounting for the effect of porosity and point defects. The model computes the conductivity of U-Si compounds based on temperature and Si molar concentration. It was fitted to the experimentally measured data for several U-Si compounds before irradiation [20-23]. The model has been used to calculate the overall thermal conductivity of a recently fabricated $\mathrm{U}_{3} \mathrm{Si}_{2}$ sample containing USi phase [24]. However, the previously reported data for $\mathrm{U}_{3} \mathrm{Si}_{2}$ was later corrected with new much lower conductivity data. To account for that change, the model is re-visited. It is realized that due to the different density of various silicides, the volumetric uranium density, which is assumed to be proportional to the electron density provided by uranium, is not the same as the uranium molar concentration. And the same is true for silicon. Therefore, the model is corrected by using the relative uranium density $\left(\rho_{u}\right)$ in reference to pure 
uranium and relative silicon density $\left(\rho_{\mathrm{si}}\right)$ to pure silicon to calculate the weighted average conductivity. Accordingly, the eq.7 in the previous report [19] is now modified as:

$$
K(c)=\frac{\rho_{u} K_{u}+\rho_{S i} K_{S i}}{1+c(1-c)\left(L_{1}+L_{2}\left(c^{2}-(1-c)^{2}\right)\right)}
$$

In eq.1, $\mathrm{K}_{\mathrm{u}}$ and $\mathrm{K}_{\mathrm{Si}}$ are the electronic conductivity of pure uranium and silicon, respectively, and they are the same as in the previous report. $\mathrm{c}$ is the molar silicon concentration. $\mathrm{L}_{1}$ and $\mathrm{L}_{2}$ are fitting parameters for the resistivity. The model in eq.1 is fitted using the data for $\mathrm{U}_{3} \mathrm{Si}$ and $\mathrm{U}_{3} \mathrm{Si}_{5}$. As shown in Fig. 4, the fitted model can predict the thermal conductivities of $\mathrm{U}_{3} \mathrm{Si}_{2}$ and USi very well, although these two compounds are not included in the fitting, showing that it is feasible to describe the conductivities of silicides based on uranium density and silicon concentrations.

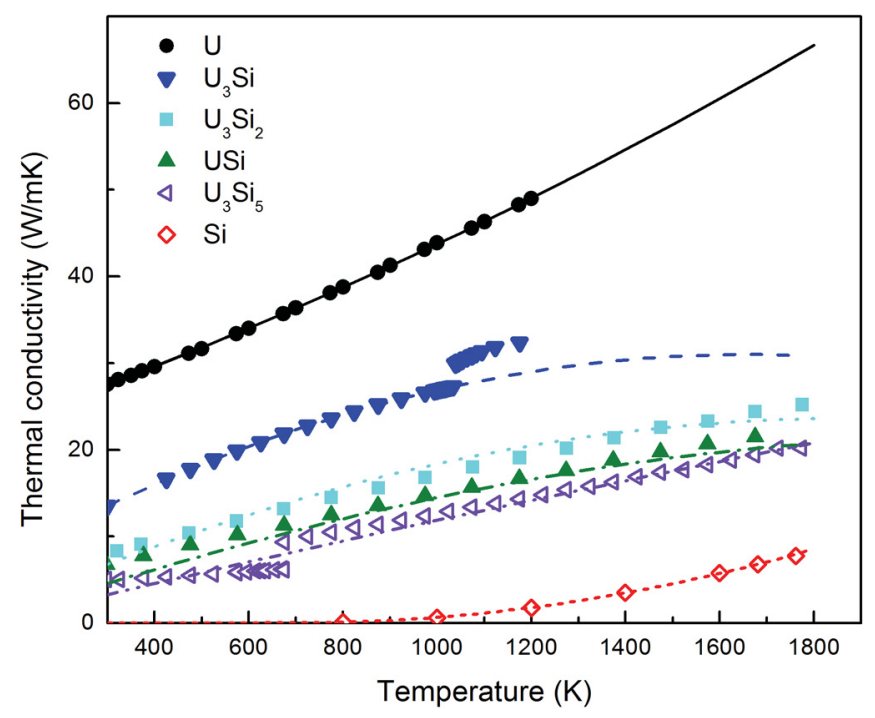

Figure 4 Thermal conductivities of $\mathrm{U}, \mathrm{U}_{3} \mathrm{Si}_{2} \mathrm{U}_{3} \mathrm{Si}_{2}, \mathrm{USi}, \mathrm{U}_{3} \mathrm{Si}_{5}$ and $\mathrm{Si}$ as functions of temperature. The curves are the model predictions using equation (1), and the symbols are experimental data. For Si only the electrical contribution is included.

\section{Summary}

In summary, under the ATF HIP project, multiscale tools have been developed including two interatomic potentials for $\mathrm{U}_{3} \mathrm{Si}_{2}$, a mesoscale model for swelling in $\mathrm{U}_{3} \mathrm{Si}_{2}$, a thermal conductivity model for U-Si compounds, and an AKMC model for $\mathrm{Cr}$ precipitation in FeCrAl. The development of these models has paved the way for studying materials behavior of $\mathrm{U}_{3} \mathrm{Si}_{2}$ and FeCrAl; such studies have previously been limited for lack of data and tools. The MEAM interatomic potential for $\mathrm{U}_{3} \mathrm{Si}_{2}$ has been utilized to calculate the surface energy, grain boundary energy, and defect production. These properties are needed for mesoscale and engineering scale fuel performance modeling. The potential is being extended to include Xe gas for studies of gas behavior in bulk and material interfaces. The swelling model in MARMOT has been used to assess swelling with burnup up to a few percent. The preliminary results suggest that the swelling is below $8 \%$ at $6 \%$ burnup. The AKMC model has been used to construct Fe-Cr-Al ternary phase diagrams at temperatures of interests, which have not previously been available. All these studies are critical for understanding the in-pile behavior of these two materials. 
Given the progress made, it should be pointed out that the current understanding of these two concepts, compared to that of $\mathrm{UO}_{2}$ and $\mathrm{Zr}$ alloys, still needs to be improved for reliable assessment of their performance. Some of the developments needed at the lower length scale have been discussed in the corresponding Sections. In addition to those, effort is suggested to be made in 1) coupling the modeling results with experiments for further development and validation and 2) integrating the lower length scale results into the engineering scale fuel performance models. 


\section{References}

1. S Bragg-Sitton. Development of advanced accident-tolerant fuels for commercial LWRS. Nuclear News, page 83, March 2014.

2. K. A. Terrani, S.J. Zinkle, and L.L. Snead. Code verification by the method of manufactured solutions. Journal of Nuclear Materials, 448:420, 2014.

3. Y. Zhang, D. Schwen, L. Aagesen, K. Ahmed, J. Yu, B. Beeler, C. Jiang, D. Andersson, Overview of lower length scale model development for accident tolerant fuels regarding U3Si2 fuel and FeCrAl cladding, INL/EXT-16-40010, Idaho National Laboratory, September 2016.

4. J. Yu, Y. Zhang, and J. Hales. Milestone report on md potential development for uranium silicide. Technical report, Idaho National Laboratory, 2016.

5. J. Yu, Y. Zhang, and J. Hales. Development of molecular dynamics potential for uranium silicide fuels. In Proceedings of Top Fuel 2016. Boise, USA, 2016.

6. B. Beeler, M. Baskes, D. Andersson, M. WD Cooper, Y. Zhang, A modified EmbeddedAtom Method interatomic potential for uranium-silicide, Journal of Nuclear Materials, Accepted, 2017.

7. L. K. Aagesen et al., Assessment of fuel swellig in U3Si2. September 2017, Idhao National Laboratory.

8. M. Plapp, Phys. Rev. E 84, 031601, 2011.

9. S.G. Kim, W.T. Kim, T. Suzuki, Phys. Rev. E 60, 7186-7197, 1999.

10. C. Jiang, Y. Zhang and E. Martinez, Lower length scale modeling of Cr precipitation in FeCrAl under thermal aging conditions. INL/EXT-17-43135, August 2017.

11. F. Soisson, A. Barbu, and G. Martin. Monte Carlo simulations of copper precipitation in dilute iron-copper alloys during thermal ageing and under electron irradiation. Acta Materialia 44:3789-3800, 1996.

12. S. Schmauder and P. Binkele. Atomistic computer simulation of the formation of cuprecipitates in steels. Computational Materials Science, 24:42-53, 2002.

13. F. Soisson and C.C. Fu. Cu-precipitation kinetics in alpha-Fe from atomistic simulations: Vacancy-trapping effects and cu-cluster mobility. Physical Review B, 76:214102, 2007.

14. E. Martinez, O. Senninger, F. Soisson, and C.C. Fu. Decomposition kinetics of Fe-Cr solid solutions during thermal aging. Physical Review B, 86:224109, 2012.

15. F. Soisson and T. Jourdan. Radiation-accelerated precipitation in $\mathrm{Fe}-\mathrm{Cr}$ alloys. Acta Materialia 103:870-881, 2016.

16. E. Vincent, C.S. Becquart, C. Pareige, P. Pareige, and C. Domain. Precipitation of the $\mathrm{FeCu}$ system: A criticle review of atomic kinetic Monte Carlo simulations. Journal of Nuclear Materials 373:387-401, 2008.

17. J. Ejenstam, M. Thuvander, P. Olsson, F. Rave, and P. Szakalos. Microstructural stability of fe-cr-al alloys at $450-550^{\circ} \mathrm{C}$. Journal of Nuclear Materials, 457:291-297, 2015.

18. S. Novy, P. Pareige, and C. Pareige. Atomic scale analysis and phase separation understanding in a thermally aged Fe-20at.\%Cr alloy. Journal of Nuclear Materials 384:96-102, 2009.

19. Y. Zhang and D. Andersson, A thermal conductivity model for U-Si compounds, LAUR-16-27736, Los Alamos, 2016. 
20. J. T. White, A. T. Nelson, D. D. Byler, J. A. Valdez, K. J. McClellan, Thermophysical properties of U3Si to $1150 \mathrm{~K}$, Journal of Nuclear Materials 452, 304-310, 2014.

21. J. T. White, A. T. Nelson, J. T. Dunwoody, D. D. Byler, D. J. Safarik, K. J. McClellan, Thermophysical properties of U3Si2 to 1773 K, Journal of Nuclear Materials 464, 275280, 2015.

22. J. T. White, A. T. Nelson, D. D. Byler, D. J. Safarik, J. T. Dunwoody, K. J. McClellan, Thermophysical properties of U3Si5 to $1773 \mathrm{~K}$, Journal of Nuclear Materials 456, 442$448,2015$.

23. J. T. White, A. T. Nelson, J. T. Dunwoody, D. D. Byler, K. J. McClellan, Thermophysical properties of USi to $1773 \mathrm{~K}$, Journal of Nuclear Materials 471, 129-135, 2016.

24. D. Antonio, K. Shrestha, J. M. Harp, C. A. Papesch, Y. Zhang, J. Carmack, and K. Gofryk, Thermal and Transport Properties of U3Si2, submitted. 